\title{
Role of crystal anisotropy on the vortex state in the Fulde-Ferrell-Larkin-Ovchinnikov (FFLO) phase
}

\author{
Vu Hung Dao, ${ }^{1}$ Dmitry Denisov, ${ }^{2}$ Alexandre Buzdin,,${ }^{3, *}$ and Jean-Pascal Brison ${ }^{4}$ \\ ${ }^{1}$ Laboratoire CRISMAT, UMR 6508, CNRS, Normandie Université, 6 Bd Maréchal Juin, 14050 Caen Cedex 4, France \\ ${ }^{2}$ Van der Waals-Zeeman Institute, University of Amsterdam, The Netherlands \\ ${ }^{3}$ Condensed Matter Theory Group, LOMA, UMR 5798, University of Bordeaux, F-33405 Talence, France \\ ${ }^{4}$ CEA Grenoble, INAC, SPSMS, F-38054 Grenoble 9, France
}

(Received 18 June 2012; revised manuscript received 26 April 2013; published 13 May 2013)

\begin{abstract}
We demonstrate that the vortex state in the Fulde-Ferrell-Larkin-Ovchinnikov (FFLO) phase may be very different depending on the field orientation relative to the crystalline axes. We numerically calculate the upper critical field near the tricritical point taking into account the modulation of the order parameter along the magnetic field as well as the higher Landau levels. For $s$-wave superconductors with the anisotropy described by an elliptical Fermi surface, we propose a general scheme for the analysis of the angular dependence of the upper critical field at all temperatures on the basis of an exact solution for the order parameter. Our results show that the transitions (with tilting magnetic field) between different types of mixed states may be a salient feature of the FFLO phase.
\end{abstract}

DOI: 10.1103/PhysRevB.87.174509

PACS number(s): 74.81.-g, 74.25.Dw, 74.25.Op

\section{INTRODUCTION}

Recent experimental studies of the superconducting state of $\mathrm{CeCoIn}_{5}$ (see Ref. 1 and references cited therein) provided evidence in favor of the Fulde-Ferrell-Larkin-Ovchinnikov (FFLO) phase existence in the high-magnetic-field region of the superconducting phase diagram. Originally, ${ }^{2,3}$ the nonuniform FFLO state has been predicted to exist in superconductors when the magnetic field is acting on the electron spins only (the case of the paramagnetic effect), but it is usually an orbital effect that is the most important, and this makes difficult the experimental observation of the FFLO phase. Moreover, the superconductor must be in the clean limit because electron scattering is detrimental for the FFLO phase in $s$-wave superconductors. ${ }^{4}$ In unconventional ( $d$-wave) superconductors, the nonuniform phase can be relatively more robust, ${ }^{5}$ but impurities eventually suppress the zero-field critical temperature. However, the orbital effect may be weakened in heavy fermion superconductors or in quasi-2D superconductors when magnetic field is applied parallel to the superconducting planes. That is why in addition to $\mathrm{CeCoIn}_{5}$ and some other heavy fermion superconductors, ${ }^{6}$ quasi-1D and quasi-2D organic superconductors are considered as good candidates for the FFLO phase realization. ${ }^{8,9}$ Recent evidence of the FFLO state has been revealed in organic quasi-2D superconductors $\lambda$-(BETS) $)_{2} \quad \mathrm{FeCl}_{4}{ }^{10}$ and $\kappa$-(BEDT-TTFS) $\mathrm{Cu}(\mathrm{NCS})_{2} .{ }^{11,12}$

In the framework of an isotropic model with $s$-wave pairing, the critical field for the FFLO phase in the presence of the orbital effect has been calculated by Gruenberg and Gunther. ${ }^{13}$ They demonstrated that the FFLO state may exist if the ratio of pure orbital effect $H_{c 2}^{\text {orb }}(0)$ and pure paramagnetic limit $H_{p}(0)$ is larger than 1.28 , i.e., the Maki parameter $\alpha_{M}=$ $\sqrt{2} H_{c 2}^{\text {orb }}(0) / H_{p}(0)$ is larger than 1.8 . The pure paramagnetic limit at $T=0$ can be estimated as $H_{p}(0)=\Delta_{0} / \sqrt{2} \mu_{B}$, where $\Delta_{0}$ is the BCS gap at $T=0$ and $\mu_{B}$ is the Bohr magneton. ${ }^{14}$ In Ref. 13, the exact solution for the order parameter was described by an FFLO modulation along the magnetic field and the zero Landau level function for the coordinates in the perpendicular plane. Further analysis ${ }^{15}$ revealed that the higher
Landau level solutions (LLS) become relevant for large values of Maki parameter $\alpha_{M}>9$ and the $H_{c 2}(T)$ curve may present regions described by different LLS. These results obtained for an isotropic model are readily generalized for the case where the electron spectrum anisotropy is described by an elliptic Fermi surface. ${ }^{16}$ In such a case, the Maki parameter becomes angular dependent and the transitions between different LLS may occur with a change of orientation of the magnetic field. For a $d$-wave pairing, the exact solution of the upper critical field is not known. However, numerical simulations and approximations with trial functions permit to study the $H_{c 2}(T)$ behavior in the FFLO phase.

It happens that for an adequate description of the FFLO state in real compounds, the form of the Fermi surface as well as the type of the superconducting pairing ( $s$-wave or $d$-wave) play a very important role because they determine the direction of the FFLO modulation. This circumstance has been demonstrated ${ }^{7,17}$ in the framework of a general phenomenological approach based on the modified GinzburgLandau (MGL) functional. ${ }^{18}$ This approach is adequate near the tricritical point (TCP) in the field-temperature phase diagram. At the TCP, the three transition lines meet: the lines separating the normal metal, the uniform superconducting state, and the FFLO state. Near the TCP, the wave vector of FFLO modulation is small and this situation may be described by the MGL functional. For the case when the deviation of the Fermi surface from the elliptical form is small, the method, ${ }^{17}$ which includes the orbital effect, permits to calculate the critical field corresponding to different LLS. Note that even in the absence of orbital effect, crystal anisotropy plays an important role since it selects the preferential direction of the FFLO modulation. It was shown that if the direction is along the magnetic field then the zero LL solution with modulation along the field is realized. On the other hand, if the preferred direction is perpendicular to the field orientation then the higher LL states should emerge without modulation along the field. In a sense, the higher LL states realize the modulation in the plain perpendicular to the field: the FFLO state in such a situation is the vortex lattice formed by higher LL solutions. 
Unfortunately, the approach ${ }^{17}$ is limited to weak deviations from the elliptic Fermi surface. In Sec. II, we develop a numerical method for the calculation of the upper critical field applicable to any cases, using a general form of the solution for the order parameter as a superposition of the different LLS. Note that the single LLS is an exact solution for the order parameter only in isotropic or quasi-isotropic (elliptic Fermi surface) $s$-wave superconductors. Otherwise, the order parameter is described by an infinite sum of the LLS. However, there is usually some dominating LL $n_{0}$ and the amplitudes of other LL rapidly decrease with an increase of $\left|n-n_{0}\right|$. Our analysis qualitatively confirms the conclusions of Ref. 17 and reveals the transitions between the FFLO states with different dominating $n_{0}$. The obtained results demonstrate that the inhomogeneous state, depending on superconductor parameters and/or magnetic field orientation, may take the form of the higher LLS with or without a modulation along the magnetic field. The transitions between these states result in a very rich dependence of the transition temperature on the magnetic field orientation.

The approach of Sec. II based on MGL is adequate near the TCP. On the other hand, the case of anisotropic $s$-wave superconductors with elliptic Fermi surface may be treated exactly at all temperatures. In Sec. III, we use the scaling transformation ${ }^{16}$ to obtain the solutions for the higher LLS in $s$-wave superconductors. As an illustration, we consider quasi-1D and quasi-2D superconductors. In quasi-2D superconductors with the in-plane orientation of the magnetic field, the higher LLS were recently studied by Shimahara. ${ }^{19}$ Note that higher LLS naturally appear in 2D superconductors in tilted magnetic field ${ }^{20,21}$ and result in the oscillatory-like angular dependence of the critical field in the FFLO state. The vortex lattices formed by such higher LLS may have very special structures different from the usual Abrikosov lattice. ${ }^{22-24}$ They may carry several flux quanta per unit cell and include vortices with different vorticity. The influence of the orbital effect on the FFLO state in superconducting films with finite thickness and parallel field orientation has been studied in Ref. 25 and the role of fluctuations at the FFLO transition was analyzed in Refs. 26-28.

\section{FORMATION OF DIFFERENT FFLO STATES UNDER THE INFLUENCE OF THE ORBITAL EFFECT}

The usual GL functional contains only the first derivatives of the order parameter, so it may be transformed by simple coordinate scaling to the isotropic form (with the corresponding renormalization of the magnetic field). That is why in the framework of the GL approach one may easily obtain the exact solution of the $H_{c 2}$ problem for any anisotropic superconductor-the order parameter is described by the $n=0$ LL function. ${ }^{29}$ The FFLO state near the TCP point can be described by MGL theory, which takes into account higher derivatives of the order parameter. In contrast to the GL functional, a coordinate rescaling cannot generally reduce the MGL functional into the isotropic form because of the higher-order derivatives (this is possible only for $s$-wave superconductivity with an elliptic Fermi surface). ${ }^{16}$ Therefore, in this section, we provide a general numerical approach for the calculation of the upper critical field. We consider a second-order transition between the normal and the FFLO state. This method is valid for both $s$-wave and $d$-wave superconductivity. Keeping in mind $\mathrm{CeCoIn}_{5}$, we will consider the case of the tetragonal symmetry.

\section{A. Modified Ginzburg-Landau theory}

To determine the critical field at a second-order transition, it is enough to expand the free energy up to the second power of the order parameter $\Psi$. Near the TCP in the paramagnetic limit the quadratic part of the MGL functional is

$$
\mathcal{F}=\Psi^{*}(\alpha+\hat{L}) \Psi
$$

with $\alpha=\alpha_{0}\left[T-T_{\mathrm{cu}}(H)\right]$, where $T_{\mathrm{cu}}(H)$ is the pure paramagnetic transition temperature between the normal and the uniform superconducting states (which would exist in the absence of the orbital effect). The differential operator $\hat{L}$ corresponds to the expansion in the order-parameter gradients, which is taken to the fourth order in order to describe the FFLO state and the tetragonal symmetry. As expected from symmetry considerations, it has the same form for $s$ - and $d$-wave order parameters, which is

$$
\begin{aligned}
\hat{L}= & -g_{x}\left(\Pi_{x}^{2}+\Pi_{y}^{2}\right)-g_{z} \Pi_{z}^{2}+\gamma_{x}\left(\Pi_{x}^{4}+\Pi_{y}^{4}\right)+\gamma_{z} \Pi_{z}^{4} \\
& +\varepsilon_{x y}\left\{\Pi_{x}^{2}, \Pi_{y}^{2}\right\}+\varepsilon_{x z}\left(\left\{\Pi_{x}^{2}, \Pi_{z}^{2}\right\}+\left\{\Pi_{y}^{2}, \Pi_{z}^{2}\right\}\right) .
\end{aligned}
$$

Here, the coordinates are chosen so that $z$ is along the crystal $c$ axis and $(x, y)$ are along the principal axes in the basal plane. The gradient operator in a magnetic field is $\Pi=$ $-i \nabla_{\mathbf{r}}+\left(2 \pi / \Phi_{0}\right) \mathbf{A}, \mathbf{A}$ is the vector potential, $\Phi_{0}$ is the flux quantum, and the anticommutator $\left\{O_{1}, O_{2}\right\}=O_{1} O_{2}+O_{2} O_{1}$. In the clean limit (see Appendix and Ref. 30), the coefficients in the $s$-wave case are

$$
\begin{gathered}
g_{i}=-\pi N_{0} K_{3}\left\langle v_{i}^{2}\right\rangle / 4, \\
\gamma_{i}=-\pi N_{0} K_{5}\left\langle v_{i}^{4}\right\rangle / 16, \\
\varepsilon_{i j}=-3 \pi N_{0} K_{5}\left\langle v_{i}^{2} v_{j}^{2}\right\rangle / 16,
\end{gathered}
$$

while for a $d$-wave order parameter $\Psi(\hat{\mathbf{k}}, \mathbf{r})=\eta(\hat{\mathbf{k}}) \Psi(\mathbf{r})$, where $\eta(\hat{\mathbf{k}}) \propto\left(k_{x}^{2}-k_{y}^{2}\right)$ and $\left\langle|\eta(\hat{\mathbf{k}})|^{2}\right\rangle=1$,

$$
\begin{gathered}
g_{i}=-\pi N_{0} K_{3}\left\langle|\eta(\hat{\mathbf{k}})|^{2} v_{i}^{2}\right\rangle / 4, \\
\gamma_{i}=-\pi N_{0} K_{5}\left\langle|\eta(\hat{\mathbf{k}})|^{2} v_{i}^{4}\right\rangle / 16, \\
\varepsilon_{i j}=-3 \pi N_{0} K_{5}\left\langle|\eta(\hat{\mathbf{k}})|^{2} v_{i}^{2} v_{j}^{2}\right\rangle / 16 .
\end{gathered}
$$

Here, $\mathbf{v}(\hat{\mathbf{k}})$ is the Fermi velocity, $N_{0}$ is the density of states at the Fermi level. The unit convention $\hbar=1$ is used. The angular brackets stand for the averaging over the Fermi surface. The coefficients

$$
K_{n}=2 T \Re \sum_{\nu=0}^{+\infty} \frac{1}{\left(\omega_{\nu}-i \mu H\right)^{n}},
$$

where $\omega_{v}=\pi T(2 v+1)$ are Matsubara frequencies and $\mu=$ $g \mu_{B} / 2$ is the electron magnetic moment. It would be interesting to calculate these coefficients for heavy fermion materials such as $\mathrm{CeCoIn}_{5}$. Considering the complicated band structure of this compound, ${ }^{31}$ the evaluation would require numerical 

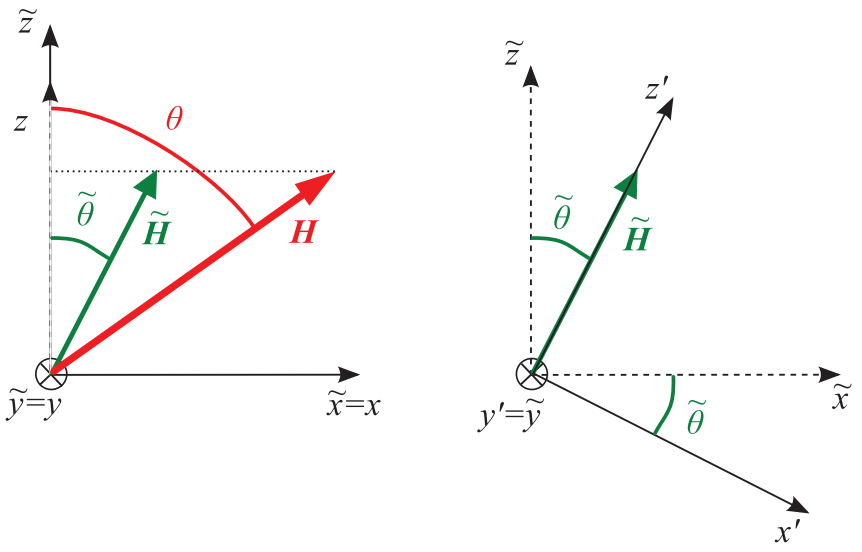

FIG. 1. (Color online) In the tetragonal symmetry, when the tensor $g_{i}$ is not isotropic, a coordinate rescaling $z \rightarrow \tilde{z}$ along the crystal $c$ axis is necessary. The rescaling introduces a renormalized field $\tilde{\mathbf{H}}$, which is not "collinear" with the actual magnetic field $\mathbf{H}$. In the rescaled coordinate system, the FFLO modulation happens along $z^{\prime}$ direction defined by $\tilde{\mathbf{H}}$.

integration from ab initio calculations, and this is beyond the scope of the present article.

In order to use the method based on LLS, the gradient expansion is transformed into a more isotropic form by rescaling coordinate $z$. For this purpose, we work within rescaled coordinates $\tilde{\mathbf{r}}=(\tilde{x}, \tilde{y}, \tilde{z})=\left(x, y, z \sqrt{g_{x} / g_{z}}\right)$, and introduce a renormalized vector potential $\tilde{\mathbf{A}}=\left(A_{x}, A_{y}, \sqrt{g_{z} / g_{x}} A_{z}\right)$ and the corresponding gradient operator $\tilde{\boldsymbol{\Pi}}=-i \nabla_{\tilde{\mathbf{r}}}+\left(2 \pi / \Phi_{0}\right) \tilde{\mathbf{A}}$. Then in the expansion, the original gradient operators $\left(\Pi_{x}, \Pi_{y}, \Pi_{z}\right)$ are replaced by $\left(\tilde{\Pi}_{x}, \tilde{\Pi}_{y}, \sqrt{g_{x} / g_{z}} \tilde{\Pi}_{z}\right)$ so that $g_{z} \Pi_{z}^{2} \rightarrow g_{x} \tilde{\Pi}_{z}^{2}$. Hence the differential operator with respect to the rescaled coordinates can be written as

$$
\begin{aligned}
\hat{L}= & -g \tilde{\Pi}^{2}+\gamma\left(\tilde{\Pi}^{2}\right)^{2}+\varepsilon_{z} \tilde{\Pi}_{z}^{4}+\frac{\varepsilon_{x}}{2}\left\{\tilde{\Pi}_{x}^{2}, \tilde{\Pi}_{y}^{2}\right\} \\
& +\frac{\tilde{\varepsilon}}{2}\left(\left\{\tilde{\Pi}_{x}^{2}, \tilde{\Pi}_{z}^{2}\right\}+\left\{\tilde{\Pi}_{y}^{2}, \tilde{\Pi}_{z}^{2}\right\}\right),
\end{aligned}
$$

where $g=g_{x}, \gamma=\gamma_{x}, \varepsilon_{z}=\left(g_{x} / g_{z}\right)^{2} \gamma_{z}-\gamma_{x}, \varepsilon_{x} / 2=\varepsilon_{x y}-$ $\gamma_{x}$, and $\tilde{\varepsilon} / 2=\left(g_{x} / g_{z}\right) \varepsilon_{x z}-\gamma_{x}$. Note that in order to recover the functional used in previous work, ${ }^{17}$ the term $\left(2 \pi / \Phi_{0}\right)^{2}\left[\tilde{\varepsilon}\left(\tilde{H}_{x}^{2}+\tilde{H}_{y}^{2}\right)+\varepsilon_{x} \tilde{H}_{z}^{2}\right]$ must be added in our expression. The latter term only shifts the energy by a constant so the solution for the order parameter is not modified.

It is important to notice that with the rescaling procedure, the renormalized field $\tilde{\mathbf{H}}=\nabla_{\tilde{\mathbf{r}}} \times \tilde{\mathbf{A}}$ is not "collinear" with the actual magnetic field $\mathbf{H}=\nabla_{\mathbf{r}} \times \mathbf{A}$ since

$$
\tilde{\mathbf{H}}=\left(\sqrt{\frac{g_{z}}{g_{x}}} H_{x}, \sqrt{\frac{g_{z}}{g_{x}}} H_{y}, H_{z}\right)
$$

(beware that they are not in the same coordinate systems). The angle $\tilde{\theta}$ that $\tilde{\mathbf{H}}$ makes with $\tilde{z}$ axis and the angle $\theta$ that $\mathbf{H}$ makes with $z$ axis (see Fig. 1) are then related by the equality

$$
\tan \tilde{\theta}=\sqrt{\frac{g_{z}}{g_{x}}} \tan \theta .
$$

As it will be discussed below, in the rescaled coordinate system $(\tilde{x}, \tilde{y}, \tilde{z})$ the FFLO order parameter is modulated along the direction of $\tilde{\mathbf{H}}$. However, when expressing back this solution with the actual coordinates $(x, y, z)$, one finds that the modulation direction makes the angle $\theta_{\text {FFLO }}$ with the $c$ axis, which is given by

$$
\tan \theta_{\mathrm{FFLO}}=\frac{g_{z}}{g_{x}} \tan \theta .
$$

We may always perform the scaling transformation and henceforth, to simplify the discussion, we may consider the case of an isotropic tensor $g_{j}=g$ with the anisotropy of the Fermi surface or the order parameter present only in the fourthorder derivatives [see Eq. (10)]. In the following of this section, we will directly operate in the rescaled coordinate system and with the renormalized magnetic field $\tilde{\mathbf{H}}$ [related to the applied field by Eq. (11)], but we will omit the tilde superscript for brevity. The coefficients $\varepsilon_{z}, \varepsilon_{x}, \tilde{\varepsilon}$ describe the deviation of the actual Fermi surface from the elliptic one and/or a pairing different from $s$-wave type. In contrast with previous work, ${ }^{17}$ they are not assumed to be small. For the appearance of the FFLO state, the coefficient $g$ must be positive otherwise, in the absence of the orbital effect, the ground state corresponds to the uniform phase.

\section{B. Numerical method}

The transition temperature is given by $T_{c}(H)=T_{\mathrm{cu}}(H)-$ $\lambda_{\min } / \alpha_{0}$, where $\lambda_{\min }$ is the smallest eigenvalue of the operator $\hat{L}$. For an arbitrary direction of the magnetic field relative to the crystal axis, the eigenfunctions of $\hat{L}$ can be looked for in the form $\Psi=\exp \left(i q z^{\prime}\right) \phi\left(x^{\prime}, y^{\prime}\right)$, where $q$ is the FFLO modulation vector along the field direction, $z^{\prime}$ is the coordinate along the field direction and $\left(x^{\prime}, y^{\prime}\right)$ are the transverse coordinates. This is because with this choice of coordinates, the operator $\Pi_{z^{\prime}}$ commutes with both $\Pi_{x^{\prime}}$ and $\Pi_{y^{\prime}}$.

In the absence of anisotropic fourth-order terms, $\phi$ can be found exactly with an adequate choice of gauge. It is one of the Landau levels $\varphi_{n}$ defined in the orthogonal $\left(x^{\prime}, y^{\prime}\right)$ plane. The eigenvalues are then

$$
\lambda^{\text {iso }}(q, n)=\gamma\left\{\left[(2 n+1) \xi_{H}^{-2}+q^{2}-q_{0}^{2}\right]^{2}-q_{0}^{4}\right\},
$$

where the LL number $n$ is a positive integer, the magnetic length is

$$
\xi_{H}=\sqrt{\frac{\Phi_{0}}{2 \pi H}}
$$

and the maximum modulation vector is

$$
q_{0}=\sqrt{\frac{g}{2 \gamma}} \text {. }
$$

In this case, $\lambda_{\min }=-\gamma q_{0}^{4}=-g^{2} / 4 \gamma$ with a degeneracy of solutions $(q, n)$. Note that the minimal eigenvalue is obtained for $(2 n+1) \xi_{H}^{-2}+q^{2}=q_{0}^{2}$, that is with high LL when $q$ is small and $n=0$ LL when $q$ is maximum. The degeneracy is lifted when anisotropic fourth-order gradient terms are present but, as shown below, the antagonistic behaviors of the LL number and the FFLO modulation vector remain.

In the general case, we diagonalize $\hat{L}$ in a (finite) subspace of functions $\varphi_{q, n}=\exp \left(i q z^{\prime}\right) \varphi_{n}\left(x^{\prime}, y^{\prime}\right)$ in order to find the smallest eigenvalue $\lambda(q)$. The latter is then minimized with respect to $q$ to get $\lambda_{\min }$ and the corresponding FFLO modulation vector $q_{c}$ at the transition. We use the orthonormal basis set composed by Landau levels $\varphi_{2 n}=\left(\hat{b}^{\dagger}\right)^{2 n} \varphi_{0} / \sqrt{(2 n) !}$ 
and $\varphi_{2 n+1}=-i\left(\hat{b}^{\dagger}\right)^{2 n+1} \varphi_{0} / \sqrt{(2 n+1) !}$. Here, $\hat{b}^{\dagger}=\frac{\xi_{H}}{\sqrt{2}}\left(\Pi_{y^{\prime}}-\right.$ $\left.i \Pi_{x^{\prime}}\right)$ is the operator of LL creation and $\varphi_{0}$ is the normalized lowest LL defined by $\hat{b} \varphi_{0}=0$. With $\hat{b}=\frac{\xi_{H}}{\sqrt{2}}\left(\Pi_{y^{\prime}}+i \Pi_{x^{\prime}}\right)$, one can easily check, for example, that $\hat{b} \hat{b}^{\dagger}-\hat{b}^{\dagger} \hat{b}=1$ and $\Pi_{x^{\prime}}^{2}+\Pi_{y^{\prime}}^{2}=\xi_{H}^{-2}\left(2 \hat{b}^{\dagger} \hat{b}+1\right)$.

As an example, we consider afterward the case when the magnetic field is rotated in $x z$ plane by an angle $\theta$ from $z$ axis. In the rotated coordinate frame, the gradient operators are transformed as $\Pi_{x}=\cos \theta \Pi_{x^{\prime}}+\sin \theta \Pi_{z^{\prime}}, \Pi_{y}=$ $\Pi_{y^{\prime}}$, and $\Pi_{z}=-\sin \theta \Pi_{x^{\prime}}+\cos \theta \Pi_{z^{\prime}}$. By expressing the operator $\hat{L}$ as a function of $\hat{b}$ and $\hat{b}^{\dagger}$, the matrix elements
$L_{m, n}=\int \varphi_{q, m}^{*} \hat{L} \varphi_{q, n}$ are found as

$$
L_{m, n}=\frac{\gamma}{\xi_{H}^{4}}\left\{\left[\left(2 n+1+k^{2}-k_{0}^{2}\right)^{2}-k_{0}^{4}\right] \delta_{m, n}+L_{m, n}^{(\varepsilon)}\right\}
$$

with

$$
k=\xi_{H} q \text { and } k_{0}=\xi_{H} \sqrt{\frac{g}{2 \gamma}} .
$$

They connect states that are separated by at most four levels. Within the above choice of basis set, the matrix is real symmetric nine-diagonal, and the non-zero terms above the diagonal are given by the anisotropic contributions

$$
\begin{aligned}
L_{n, n}^{(\varepsilon)}= & \frac{\varepsilon_{z}}{4 \gamma}\left[4 c^{4} k^{4}+12 c^{2} s^{2}(2 n+1) k^{2}+3 s^{4}\left(2 n^{2}+2 n+1\right)\right] \\
& +\frac{\tilde{\varepsilon}}{4 \gamma}\left[s^{2}\left(3 c^{2}-1\right)+2 s^{2}\left(1+3 c^{2}\right)\left(n^{2}+n\right)+2\left(1+c^{2}-6 c^{2} s^{2}\right)(2 n+1) k^{2}+4 c^{2} s^{2} k^{4}\right] \\
& +\frac{\varepsilon_{x}}{4 \gamma}\left[c^{2}\left(2 n^{2}+2 n-1\right)+2 s^{2}(2 n+1) k^{2}\right], \\
L_{n, n+1}^{(\varepsilon)}= & (-1)^{n} \sqrt{n+1} k \sin 2 \theta\left\{\frac{\varepsilon_{z}}{\gamma}\left(\frac{3}{\sqrt{2}} s^{2}(n+1)+\sqrt{2} c^{2} k^{2}\right)-\frac{\varepsilon_{x}(n+1)}{2 \sqrt{2} \gamma}+\frac{\tilde{\varepsilon}}{\sqrt{2} \gamma}\left[\frac{1+3 \cos 2 \theta}{2}(n+1)-k^{2} \cos 2 \theta\right]\right\}, \\
L_{n, n+2}^{(\varepsilon)}= & \sqrt{(n+2) ! / n !} s^{2}\left\{\frac{\varepsilon_{x}}{2 \gamma} k^{2}-\frac{\varepsilon_{z}}{\gamma}\left[\left(n+\frac{3}{2}\right) s^{2}+3 c^{2} k^{2}\right]+\frac{\tilde{\varepsilon}}{2 \gamma}\left[-c^{2}(2 n+3)+\left(6 c^{2}-1\right) k^{2}\right]\right\}, \\
L_{n, n+3}^{(\varepsilon)}= & (-1)^{n} \sqrt{(n+3) ! / n !} \frac{k \sin 2 \theta}{\sqrt{2}}\left[\left(\frac{\tilde{\varepsilon}}{\gamma}-\frac{\varepsilon_{z}}{\gamma}\right) s^{2}-\frac{\varepsilon_{x}}{2 \gamma}\right], \\
L_{n, n+4}^{(\varepsilon)}= & \sqrt{(n+4) ! / n !} \frac{1}{4}\left[\left(\frac{\varepsilon_{z}}{\gamma}-\frac{\tilde{\varepsilon}}{\gamma}\right) s^{4}-\frac{\varepsilon_{x}}{\gamma} c^{2}\right],
\end{aligned}
$$

with $s=\sin \theta$ and $c=\cos \theta$

\section{Angle dependence of the critical field and of the structure of the FFLO state}

A previous work ${ }^{17}$ showed that due to the effect of anisotropy three different types of solution for the inhomogeneous state can be realized: (a) the maximum FFLO modulation occurs along the magnetic field with the zero Landau level state, (b) both modulation and higher Landau level state, and (c) the highest possible Landau level and no modulation along the field (or a modulation with a very small wave vector). Moreover, due to the specific form of the Fermi surface, a variation of magnetic field orientation may provoke transitions between states with different Landau levels. However, a single-level approximation was used to get analytical results for these solutions. Due to this approximation, the analytical results were valid for magnetic fields higher than $H \gg \Phi_{0} \frac{g}{\gamma} \sqrt{\frac{\varepsilon}{\gamma}}$ where $\varepsilon=\max \left(\left|\varepsilon_{z}\right|,\left|\varepsilon_{x}\right|,|\tilde{\varepsilon}|\right)$. In the present work, we show using numerical calculations, that taking into consideration the full set of Landau levels, the results qualitatively remain true for arbitrary values of the magnetic field $H$.

We calculate the transition temperature and the corresponding FFLO state when the magnetic field is applied in $x z$ plane. Typical results are displayed in Fig. 2 where we have set $\varepsilon_{x}=\tilde{\varepsilon}=0$. The form of the FFLO solution depends only on the parameter ratios $\varepsilon_{z} / \gamma$ and $\xi_{H} \sqrt{\frac{g}{2 \gamma}}$ [see, e.g., expression (17) of the operator $\hat{L}$ in the basis of LL]. As illustrated in Fig. 2(a), the FFLO state can appear with the FFLO modulation vector $q_{c}$ equal to the maximum value $q_{0}$ and the $n=0$ LL when the field is along $z$ axis. In contrast, for $H$ along $x$ axis, there is no longitudinal modulation and the solution is composed by higher LL, which results in transverse modulations of the order parameter. In the isotropic case, the opposite variations of $q_{c}$ and of the LL number are easily explained because $(2 n+1) \xi_{H}^{-2}+q_{c}^{2}$ is equal to $q_{0}^{2}$ [see Eq. (14)]. Our numerical solutions show that anisotropy does not modify these antagonistic behaviors. When the field is rotated from one axis to the other, the state at the transition is transformed with a continuous variation of the FFLO modulation and a smooth evolution of its expansion over the LL. However, for smaller values of $\xi_{H} q_{0}$ or $\varepsilon_{z} / \gamma$, the variation with the field orientation can be discontinuous with jumps of vector $q_{c}$ [see Figs. 2(b) and 2(c)] and of the average LL. The discontinuities occur when for the same field there are several local minima of $\lambda(q)$ or, equivalently, local maxima of transition temperature $T_{c}(q)=T_{\mathrm{cu}}-\lambda(q) / \alpha_{0}$. When rotating the field, the critical temperature $T_{c}=\max T_{c}(q)$ can change 
(a)
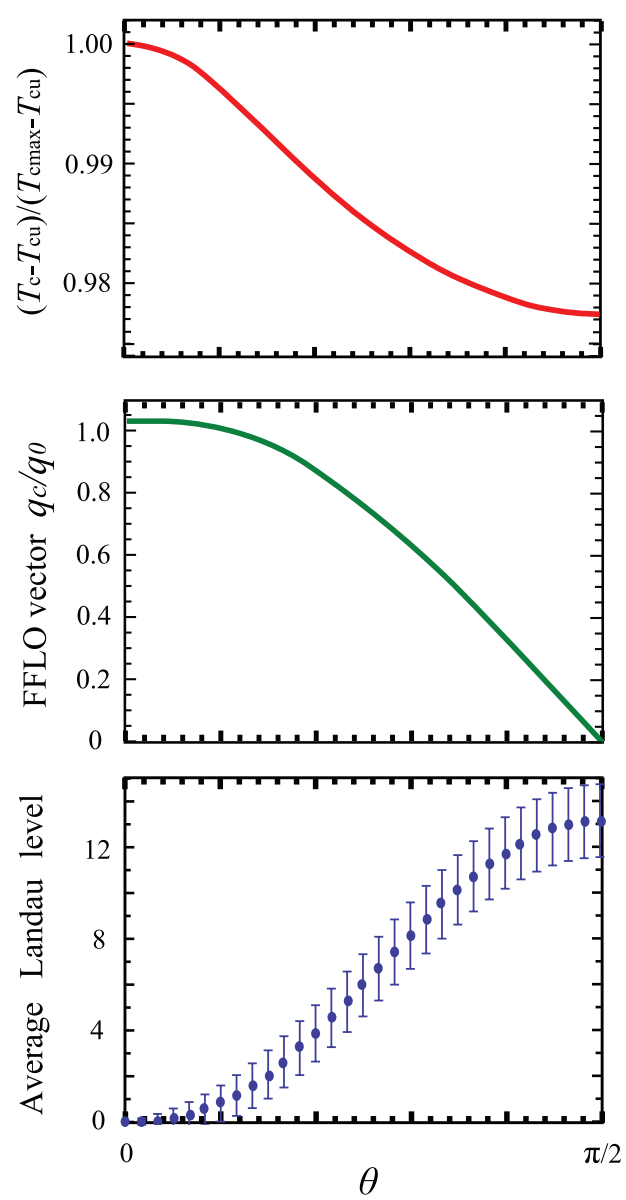

(b)
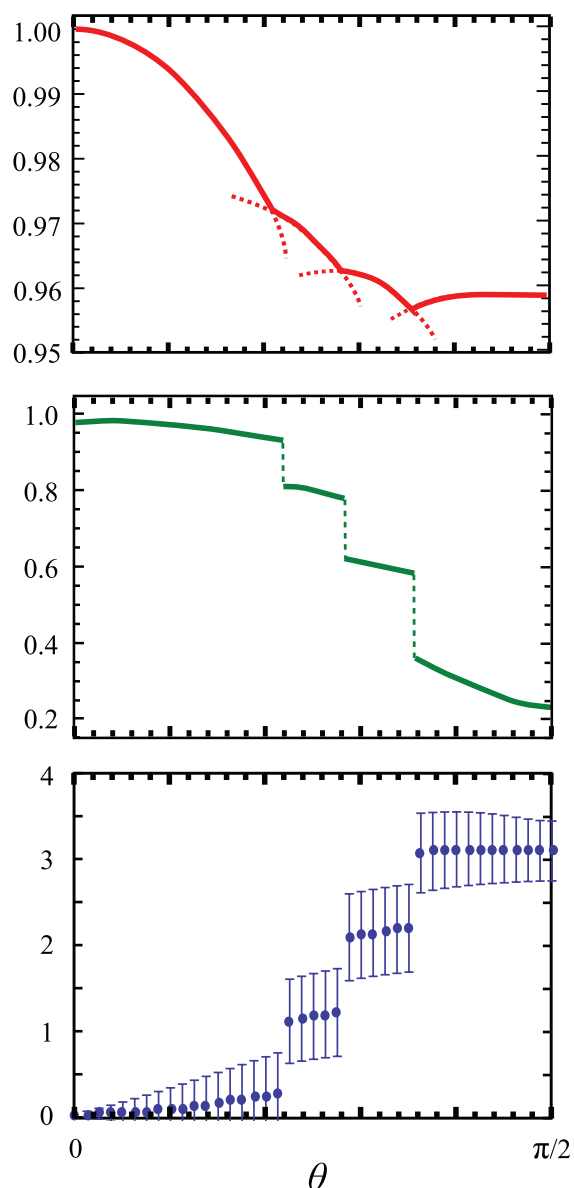

(c)
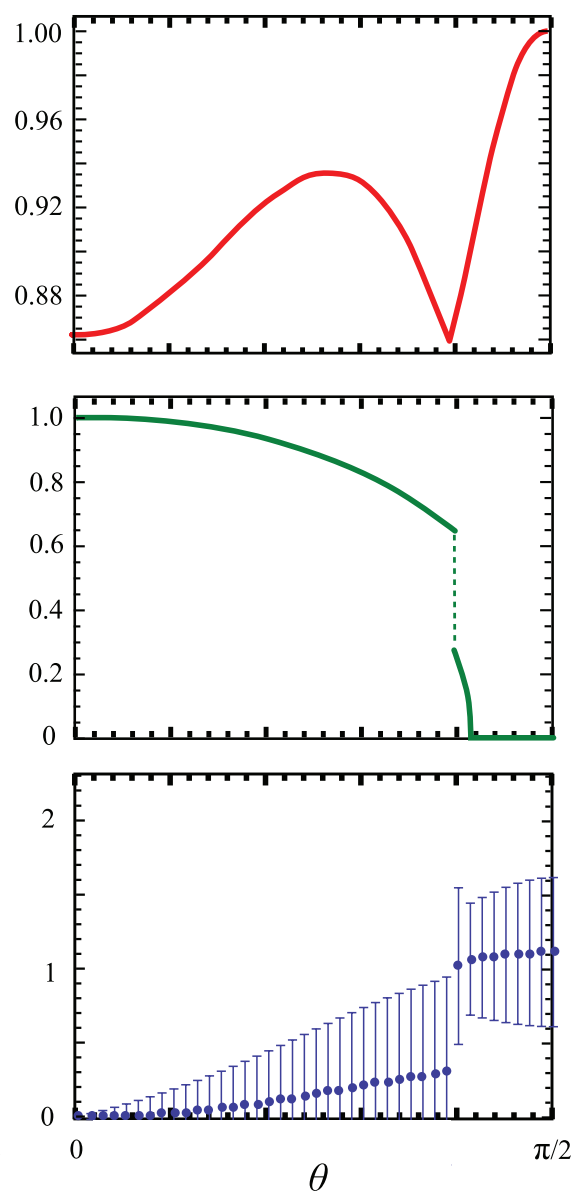

FIG. 2. (Color online) Angle dependence of the critical temperature and of the corresponding FFLO state for parameters $\varepsilon_{x}=\tilde{\varepsilon}=0$, with (a) $\varepsilon_{z}=-0.1 \gamma, g=50 \gamma \xi_{H}^{-2}$, (b) $\varepsilon_{z}=-0.1 \gamma, g=15 \gamma \xi_{H}^{-2}$, and (c) $\varepsilon_{z}=-0.5 \gamma, g=4 \gamma \xi_{H}^{-2}$. In the top plots, the critical temperature (solid line) is obtained as the maximum of the temperatures of transition into states with different FFLO modulation vectors (dotted lines). The vertical bars in the bottom plots show the mean-square deviation of the Landau levels composing the state from the average LL.

from one local maximum to another, and this is realized with a jump of the modulation vector $q_{c}$. These sharp transitions are then manifested by kinks between bumps in the angle dependence of $T_{c}$. Figure 3(a) shows that, for other anisotropy parameters, the jump can occur between states separated by more than one LL. As expected from the condition of single-level approximation $H \gg \Phi_{0} \frac{g}{\gamma} \sqrt{\frac{\varepsilon}{\gamma}}$ or equivalently $\xi_{H}^{2} q_{0}^{2} \sqrt{\frac{\varepsilon}{\gamma}} \ll 1$, the number of LL that contribute significantly in the expansion of the FFLO state increases with the inverse of the field and/or the anisotropy (see Fig. 4). The broadening of the expansion over the LL ends up in suppressing the discontinuities. In addition, as illustrated in Figs. 3(b) and 3(c), the FFLO modulation and the transition temperature can vary nonmonotonously with the field angle. It is interesting to note that at the angles when the average LL is maximum the wave vector of modulation is minimal (and vice versa) and it can even drop to zero [see Fig. 3(c)]. At these regions, the inhomogeneous state corresponds to the highest LL states only. The experimental observation of such a nontrivial angular dependence of $T_{c}$ would be a strong evidence of the FFLO state.

\section{HIGHER LANDAU LEVEL STATES IN THE FRAMEWORK OF THE MODEL OF EFFECTIVE MASS ANISOTROPY}

In this section, we demonstrate how the higher LLS naturally appear in the exactly solvable microscopic model of the FFLO transition in $s$-wave superconductors in a framework of anisotropic effective mass model. As it has been already noted in the case of the pure paramagnetic limit, this model is reduced by a scaling transformation to the isotropic one with an arbitrary direction of the FFLO modulation. ${ }^{16}$ In the presence of the orbital effect, the situation is different and we consider here the uniaxial anisotropy (note that our results are readily generalized to the arbitrary anisotropy case). We are interested by a part of the Hamiltonian depending on the magnetic field $\mathcal{H}_{\text {orb }}+\mathcal{H}_{\text {Pauli }}$ with

$$
\begin{gathered}
\mathcal{H}_{\text {orb }}=-\frac{1}{2 m}\left(\frac{\partial}{\partial x}-\frac{i e}{c} y H \cos \theta\right)^{2}-\frac{1}{2 m}\left(\frac{\partial}{\partial y}\right)^{2} \\
-\frac{1}{2 m_{z}}\left(\frac{\partial}{\partial z}-\frac{i e}{c} y H \sin \theta\right)^{2}, \\
\mathcal{H}_{\text {Pauli }}=\mu_{B} H \sigma_{z},
\end{gathered}
$$


(a)
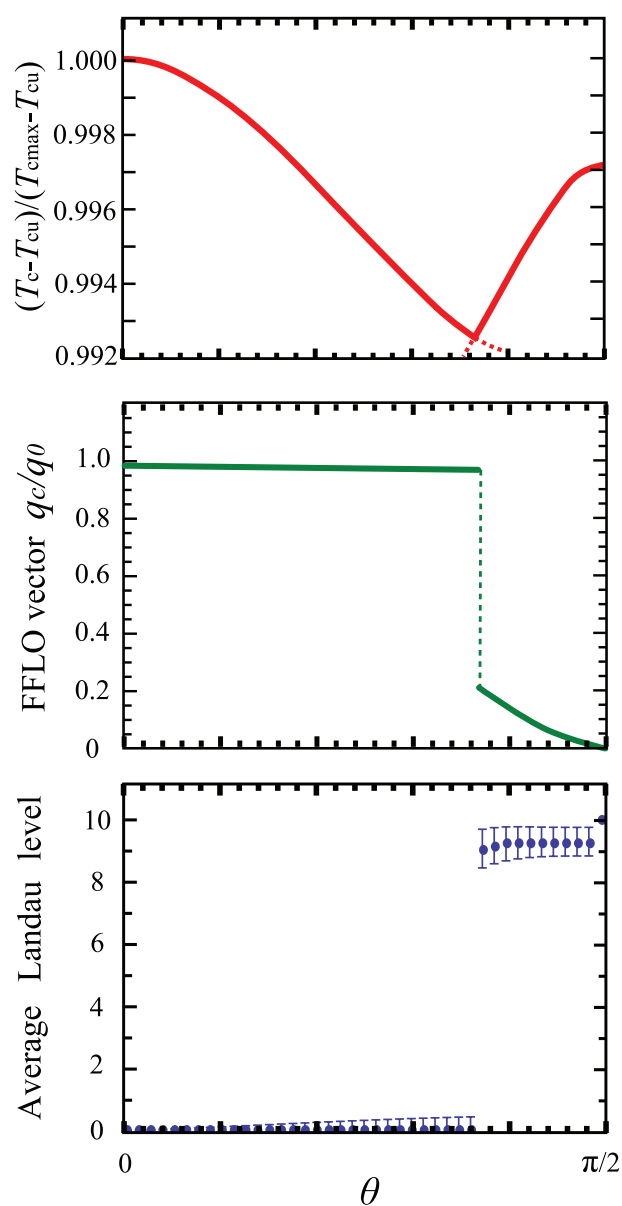

(b)
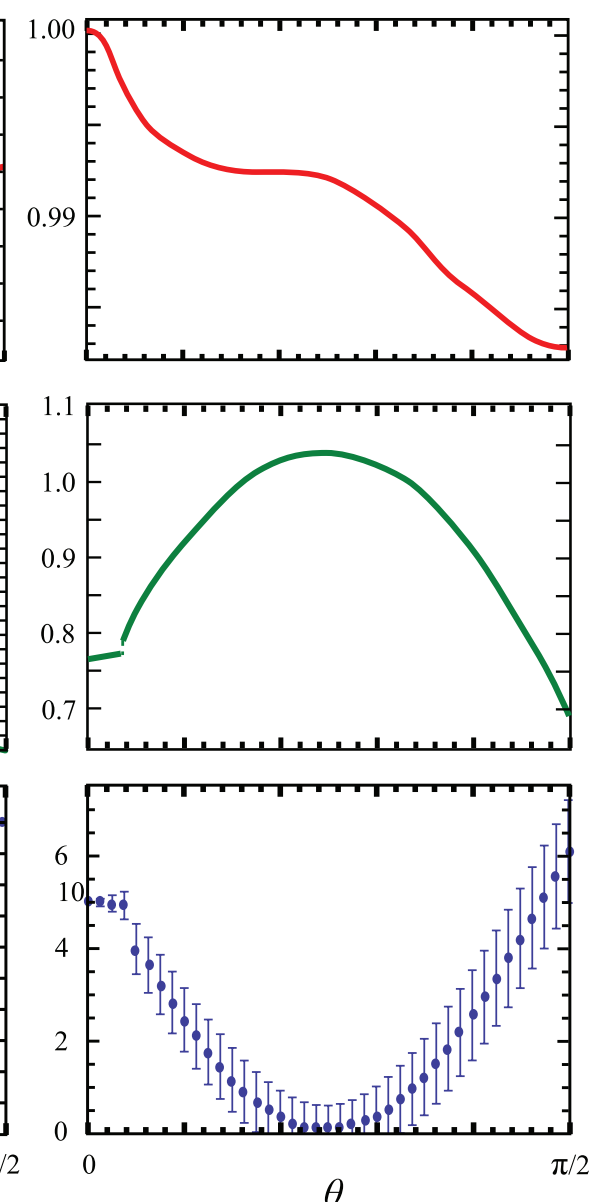

(c)
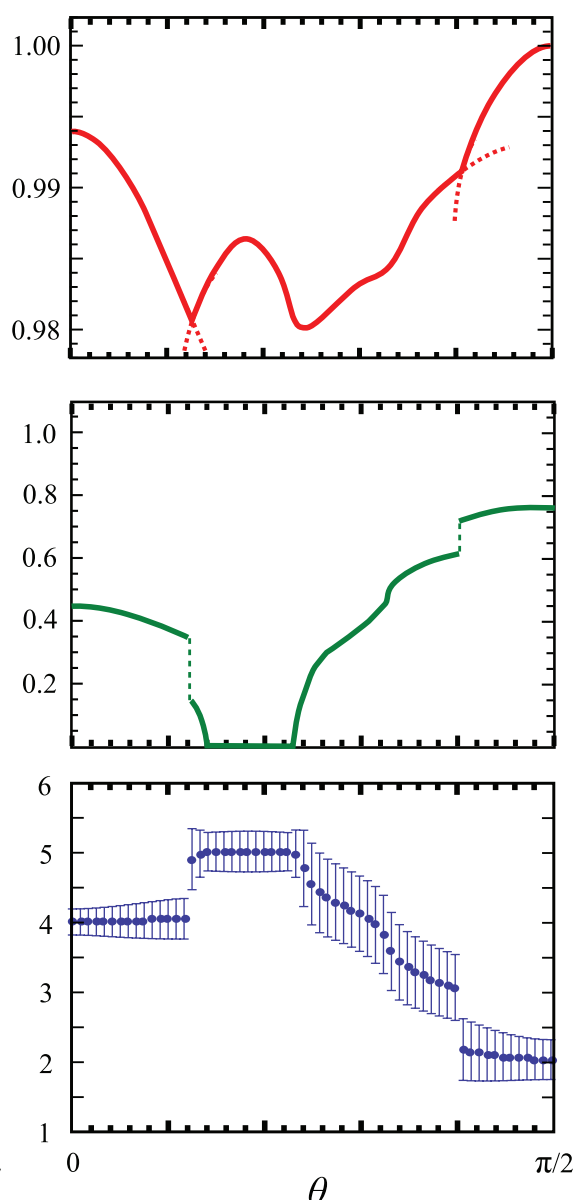

FIG. 3. (Color online) Angle dependence of the critical temperature and of the corresponding FFLO state for parameters (a) $\tilde{\varepsilon}=\varepsilon_{z}=0$, $\varepsilon_{x}=0.5 \gamma, g=40 \gamma \xi_{H}^{-2}$, (b) $\varepsilon_{x}=\varepsilon_{z}=0, \tilde{\varepsilon}=-0.5 \gamma, g=40 \gamma \xi_{H}^{-2}$, and (c) $\tilde{\varepsilon}=\varepsilon_{x}=-0.3 \gamma, \varepsilon_{z}=0.2 \gamma, g=20 \gamma \xi_{H}^{-2}$. Same conventions as in Fig. 2.

where we consider the effective mass $m_{x}=m_{y}=m$ and the magnetic field $\mathbf{H}$ is in $x z$ plane making an angle $\theta$ with $z$ axis. Our treatment can be readily generalized to the case of an anisotropic $g$ factor. ${ }^{16}$ The gauge of the vector potential $\mathbf{A}$ is chosen as $A_{x}=y H \cos \theta, A_{y}=0, A_{z}=y H \sin \theta$ and the spin quantization axis is along the magnetic field.

Performing the scaling transformation $z=\tilde{z} \sqrt{\frac{m}{m_{z}}}$ the orbital part becomes $^{16}$

$$
\begin{aligned}
\mathcal{H}_{\text {orb }}= & -\frac{1}{2 m}\left(\frac{\partial}{\partial x}-\frac{i e}{c} y H \cos \theta\right)^{2}-\frac{1}{2 m}\left(\frac{\partial}{\partial y}\right)^{2} \\
& -\frac{1}{2 m}\left(\frac{\partial}{\partial \tilde{z}}-\frac{i e}{c} y H \sqrt{\frac{m}{m_{z}}} \sin \theta\right)^{2},
\end{aligned}
$$

i.e., it corresponds to the isotropic metal with an effective mass $m$ at the orbital magnetic field $\widetilde{H}=H \sqrt{\cos ^{2} \theta+\frac{m}{m_{z}} \sin ^{2} \theta}$ ( $\widetilde{H}_{z}=H_{z}$ and $\widetilde{H}_{x}=H_{x} \sqrt{\frac{m}{m_{z}}}$ ). The Pauli contribution may be written as

$$
\mathcal{H}_{\text {Pauli }}=\mu_{B} H \sigma_{z}=\frac{\mu_{B} \tilde{H} \sigma_{z}}{\sqrt{\cos ^{2} \theta+\frac{m}{m_{z}} \sin ^{2} \theta}}=\tilde{\mu}_{B} \tilde{H} \sigma_{z}
$$

with the angular dependent effective Bohr magneton $\widetilde{\mu}_{B}(\theta)=$ $\mu_{B} / \sqrt{\cos ^{2} \theta+\frac{m}{m_{z}} \sin ^{2} \theta}$.

In fact, we have reduced the problem of the FFLO critical field calculation to that of the isotropic model with the field $\widetilde{H}$ and the effective Bohr magneton $\widetilde{\mu}_{B}(\theta)$. The corresponding Maki parameter is $\alpha_{M}=\sqrt{2} H_{c 2}^{\text {orb }}(0) / H_{p}(0)$ with, in our case, $H_{c 2}^{\text {orb }}(0)$ that is determined by the effective mass $m$ and then is the pure orbital field along $z$ axis, while $H_{p}(0)=\frac{\Delta_{0}}{\sqrt{2} \widetilde{\mu}_{B}(\theta)}$. So the Maki parameter becomes angular dependent:

$\alpha_{M}(\theta)=\frac{2 \mu_{B} H_{c 2}^{z \text { orb }}(0)}{\Delta_{0} \sqrt{\cos ^{2} \theta+\frac{m}{m_{z}} \sin ^{2} \theta}}=0.54\left[\frac{d H_{c 2}(\theta)}{d T}\right]_{T=T_{c 0}} \frac{\mathrm{K}}{\mathrm{T}}$.

Remarkably, in the latter expression for $\alpha_{M}(\theta)$ enters only the slope of $H_{c 2}$ at the same angle $\theta$. As it was demonstrated in Ref. 15 for large values of the Maki parameter, $\alpha_{M}>9$, the critical FFLO field at low temperature is determined by higher LLS. In the case of a large quasi-2D anisotropy $\left(\frac{m_{z}}{m} \gg 1\right)$, this situation is realized when the Maki parameter is strongly increased as the field is tilted near $x y$ plane. On the contrary, 


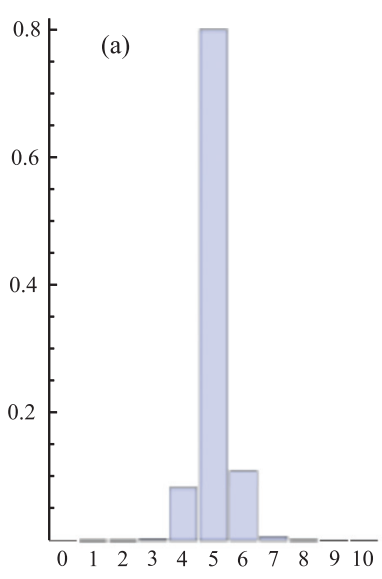

(b)

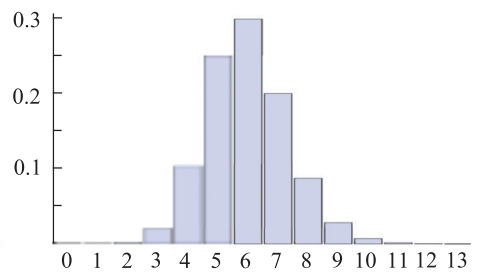

(c)

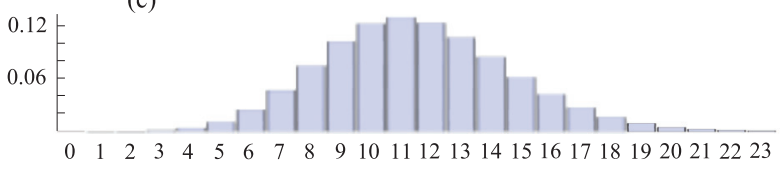

FIG. 4. (Color online) Weight $\left|c_{n}\right|^{2}$ of the $n$th Landau level $\varphi_{n}$ in the expansion of the FFLO state $\Psi=\exp (i q z) \sum_{n} c_{n} \varphi_{n}$ at the field angle $\theta=\pi / 4$, for parameters $\varepsilon_{x}=\tilde{\varepsilon}=0, g=50 \gamma \xi_{H}^{-2}$, with (a) $\varepsilon_{z}=-0.01 \gamma$, (b) $\varepsilon_{z}=-0.1 \gamma$, and (c) $\varepsilon_{z}=-0.5 \gamma$.

for the quasi-1D anisotropy $\left(\frac{m_{z}}{m} \ll 1\right)$, the Maki parameter is maximum for the field orientated along $z$ axis.

The critical field may be numerically calculated from the formula $^{15}$

$$
\begin{aligned}
\ln \left(\frac{T}{T_{\mathrm{co}}}\right)= & \frac{T}{T_{\mathrm{co}}} 2 \pi \operatorname{Re} \sum_{\nu=0}^{+\infty}\left[(-1)^{n} \int \frac{\beta L_{n}(2 \beta y)}{\sqrt{\widetilde{q}^{2}+y}}\right. \\
& \left.\times \tan ^{-1}\left(\frac{T_{\mathrm{co}}}{\omega_{\nu}+i \mu_{B} H}\right) e^{-\beta y} d y-\frac{T_{\mathrm{co}}}{\omega_{n}}\right],
\end{aligned}
$$

where $T_{\text {co }}$ is the (zero-field) critical temperature, $\omega_{v}=$ $\pi T(2 v+1)$ are the Matsubara frequencies, $L_{n}$ are Laguerre polynomials, and

$$
\beta=\frac{T_{\mathrm{co}}}{H(\theta)} \frac{7 \zeta(3)}{12 \pi^{2}}\left[\frac{d H_{c 2}(\theta)}{d T}\right]_{T=T_{c 0}} .
$$

The LL number $n$ and the dimensionless vector of the FFLO modulation $\widetilde{q}=\hbar v_{F} q / 2 T_{\text {co }}$ are chosen in a way to give the maximum critical field $H(\theta)$.

The calculated values of the upper critical field at $T=0 \mathrm{~K}$ as a function of the critical field slope at $T=T_{\text {co }}$ are presented in Fig. 5. The LLS with $n>0$ appear at $-\left(\frac{d H_{c 2}(\theta)}{d T}\right)_{T=T_{c 0}}>$ $18 \mathrm{~T} / \mathrm{K}$. We see that with an increase of the slope the Landau level number $n$ increases, while the FFLO modulation vector drops. For some slopes, it occurs to be zero, and then the inhomogeneous state is purely higher LLS. In the upper panel of Fig. 5, we observe the nonmonotonous behavior of the upper critical field as a function of the slope (or the anisotropy $\frac{m_{z}}{\mathrm{~m}}$ ). With the increase of the slope, the orbital effect is switched off and we approach the pure paramagnetic limit for the 3D case. However, at $T=0 \mathrm{~K}$, the transition into FFLO state is a first-order transition ${ }^{32}$ and then the calculated upper critical field should be the overcooling field of the normal phase.

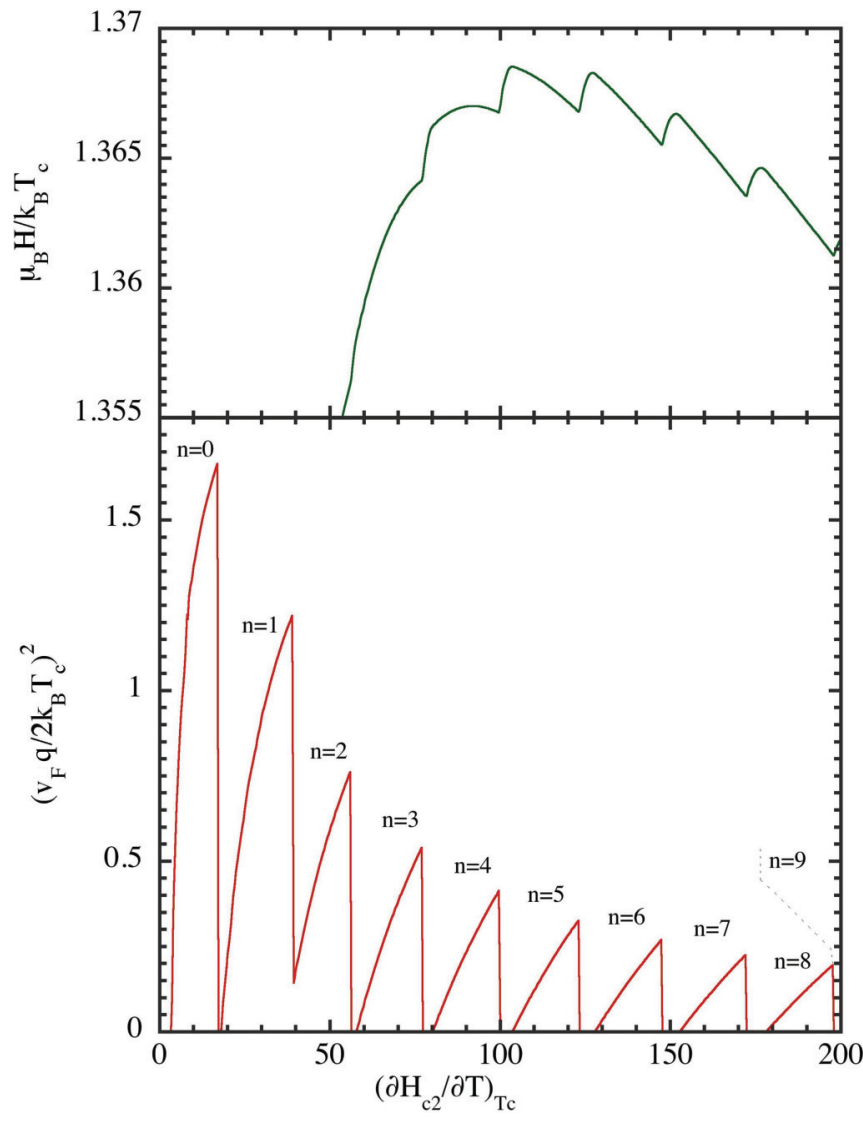

FIG. 5. (Color online) (Top) Zero-temperature critical field as a function of the initial slope $\left(\frac{d H_{c 2}}{d T}\right)_{T=T_{c 0}}$. The transitions between the higher LLS are clearly seen. (Below) FFLO modulation vector.

Large values of the Maki parameter suitable for the observation of these higher LLS are mainly expected in strongly (quasi-2D or quasi-1D) anisotropic systems. In such systems, the formation of the higher LLS may be clearly observed on the angular dependence of the critical field, which will reproduce the dependence on the initial slope displayed on Fig. 5. In Fig. 6, such an angular dependence is presented for a maximum slope $-\left[\frac{d H_{c 2}\left(90^{\circ}\right)}{d T}\right]_{T=T_{c 0}}=17 \mathrm{~T} / \mathrm{K}$, with a ratio of effective masses $\frac{m_{z}}{m}=100$, below the threshold of higher LLS formation. We see in Fig. 6 the standard behavior inherent to the anisotropic mass model. The situation is very different in Fig. 7, where the slope $-\left[\frac{d H_{c 2}\left(90^{\circ}\right)}{d T}\right]_{T=T_{c 0}}=60 \mathrm{~T} / \mathrm{K}$ is well above the threshold. At low temperature, the angular dependence $H_{c 2}(\theta)$ clearly reveals the transition between the higher LLS, making the overall shape of the $H_{c 2}(\theta)$ curve very peculiar, and somewhat similar to the corresponding results of Sec. II. Note that rounding of the angular dependence $H_{c 2}(\theta)$ in the FFLO phase was also observed in $\mathrm{CeCoIn}_{5} / \mathrm{YbCoIn}_{5}$ superlattices ${ }^{33}$ where a CeCoIn 5 layer of three-unit-cell thickness may be considered as a 2D superconductor.

Note that in isotropic systems the FFLO modulation vector $\mathbf{q}$ is directed along the applied magnetic field. ${ }^{13,14}$ In an anisotropic superconductor, the FFLO modulation is described by $\exp \left[i q\left(\frac{\widetilde{H}_{z}}{\tilde{H}} \tilde{z}+\frac{\widetilde{H}_{x}}{\tilde{H}} x\right)\right] \sim$ $\exp \left[i q\left(\frac{H \cos \theta}{\widetilde{H}} \sqrt{\frac{m_{z}}{m}} z+\sqrt{\frac{m}{m_{z}}} \frac{H \sin \theta}{\widetilde{H}} x\right)\right], \quad$ that is, 


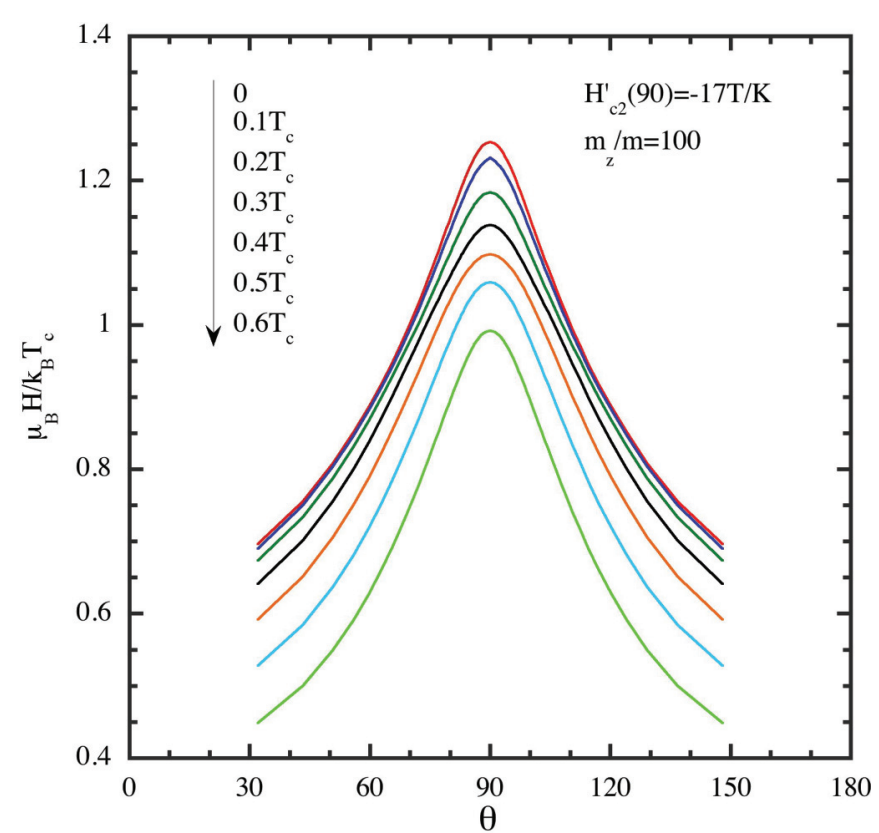

FIG. 6. (Color online) The angular dependence of the upper critical field $H_{c 2}(\theta)$ at different temperature for the initial slope $-\left(\frac{d H_{c 2}\left(90^{\circ}\right)}{d T}\right)_{T=T_{c 0}}=17 \mathrm{~T} / \mathrm{K}$. This case corresponds to the $n=0 \mathrm{LL}$ state.

$\sim \exp \left[\frac{i q}{\sqrt{\cos ^{2} \theta+\frac{m}{m_{z}} \sin ^{2} \theta}}\left(z \cos \theta+\frac{m}{m_{z}} x \sin \theta\right)\right]$.

Therefore

the angle $\theta_{\mathrm{FFLO}}$ that the direction of the FFLO modulation makes with $z$ axis is given by

$$
\tan \theta_{\mathrm{FFLO}}=\frac{m}{m_{z}} \tan \theta .
$$

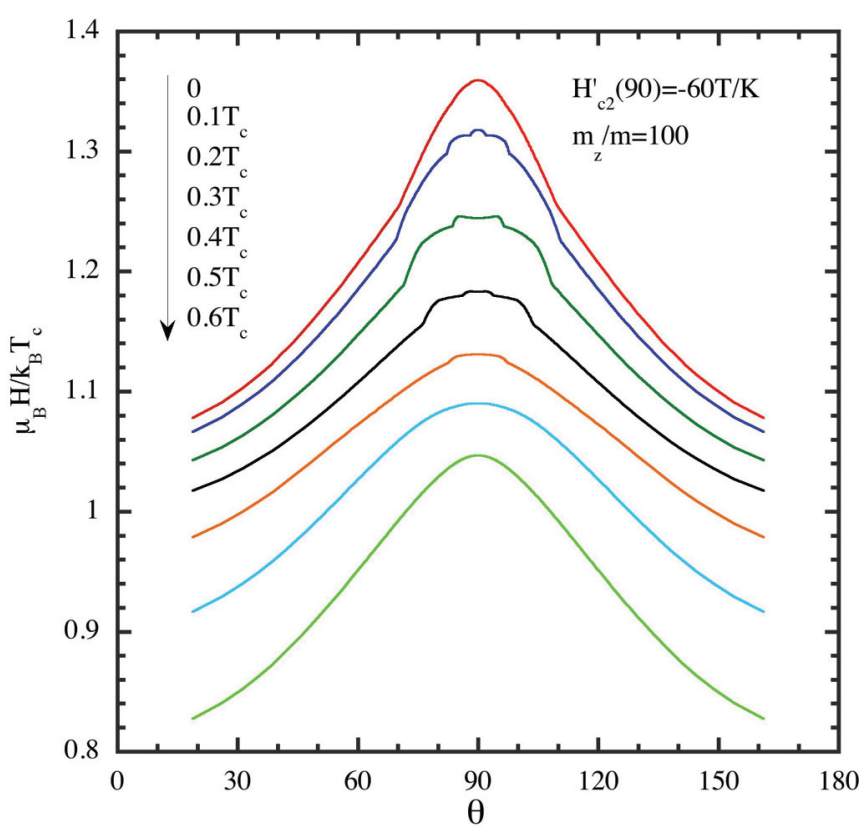

FIG. 7. (Color online) The angular dependence of the upper critical field $H_{c 2}(\theta)$ at different temperature for the initial slope $-\left[\frac{d H_{c 2}\left(90^{\circ}\right)}{d T}\right]_{T=T_{c 0}}=60 \mathrm{~T} / \mathrm{K}$. At low temperature, the transitions between the different LLS are responsible for the peculiar form of $H_{c 2}(\theta)$ dependence.
This means that for quasi-2D anisotropy the FFLO modulation vector deviates from the field direction toward $z$ axis, while for the quasi-1D anisotropy it lies closer to $x y$ plane.

The appearance of the higher LLS in quasi-2D superconductors when the magnetic field direction approaches the $x y$ plane is consistent with the prediction of such states in 2D superconductors in a tilted magnetic field. ${ }^{20,21,34}$ Note that some organic superconductors are strongly anisotropic and, for example, in $\lambda$-(BETS $)_{2} \mathrm{FeCl}_{4}$ the slope $-\left[\frac{d H_{c 2}\left(90^{\circ}\right)}{d T}\right]_{T=T_{c 0}} \sim$ $18 \mathrm{~T} / \mathrm{K}$ was measured. ${ }^{35}$ At the same time, the slope $60 \mathrm{~T} / \mathrm{K}$ (see Fig. 7) practically corresponds to 2D superconductivity.

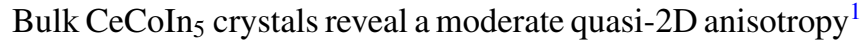
and we believe that the details of the Fermi surface structure should play an important role for the higher LL states formation (see Sec. II).

\section{CONCLUSIONS}

In real compounds, crystal structure plays a dominant role in determining the type of FFLO state. Of course, it will also influence the vortex structure. The FFLO state may be characterized by an unidimensional modulation of the order parameter, and/or by the emergence of higher Landau level states. This is a crucial difference with superconductivity without FFLO state, where the crystal structure influences only the type of Abrikosov vortex lattice. The higher Landau level FFLO states should be realized in systems with strong uniaxial anisotropy and near the in-plane orientation of the magnetic field. In such a case, the higher Landau level states should lead to an unusual angular dependence of $H_{c 2}$ with a cascade of transitions between higher Landau level states. Our approach based on the MGL theory is valid near the tricritical point (otherwise higher order derivatives start to play an important role). However, it shows that the higher Landau level states should be taken into account for the adequate description of the FFLO phase at all temperatures in real compounds.

\section{ACKNOWLEDGMENTS}

The authors are grateful to A. Melnikov and A. Samokhvalov for useful discussions and comments. This work was supported by the French ANR project SINUS and Electrovortex and European IRSES program SIMTECH (Contract No. 246937).

\section{APPENDIX: MICROSCOPIC EXPRESSION OF THE GRADIENT COEFFICIENTS IN THE MGL FUNCTIONAL}

The expression for the MGL functional has been derived from a microscopic theory at finite temperature in the vicinity of TCP in Ref. 30. The case of a metal with arbitrary concentration of pointlike impurities (i.e., $s$-wave scattering) has been considered. For $s$-wave pairing superconductivity, the functional quadratic in the order parameter $\Psi$ is

$$
\begin{aligned}
\mathcal{F}= & \Psi^{*}\left\{\alpha_{s}+\pi N_{0}\left[\frac{K_{21}}{4}\left\langle(\mathbf{v} \cdot \boldsymbol{\Pi})^{2}\right\rangle-\frac{K_{23}}{16}\left\langle(\mathbf{v} \cdot \boldsymbol{\Pi})^{4}\right\rangle\right.\right. \\
& \left.\left.-\frac{K_{33}}{32 \tau}\left\langle(\mathbf{v} \cdot \boldsymbol{\Pi})^{2}\right\rangle^{2}\right]\right\} \Psi .
\end{aligned}
$$


Here, $\mathbf{v}(\hat{\mathbf{k}})$ is the Fermi velocity, $N_{0}$ is the density of states at the Fermi level, and $\tau$ is the mean-free time of quasiparticle scattering. The unit convention $\hbar=1$ is used. The angular brackets stand for the averaging over the Fermi surface. The coefficients

$$
K_{n m}=2 T \Re \sum_{\nu=0}^{+\infty} \frac{1}{\left(\omega_{v}-i \mu H\right)^{n}\left(\tilde{\omega}_{v}-i \mu H\right)^{m}},
$$

where $\omega_{v}=\pi T(2 v+1)$, are the Matsubara frequencies, $\tilde{\omega}_{v}=$ $\omega_{\nu}+\operatorname{sign}\left(\omega_{\nu}\right) / 2 \tau$, and $\mu=g \mu_{B} / 2$ is the electron magnetic moment.
For a $d$-wave superconductor with an order parameter $\Psi(\hat{\mathbf{k}}, \mathbf{r})=\eta(\hat{\mathbf{k}}) \Psi(\mathbf{r})$, where $\eta(\hat{\mathbf{k}}) \propto\left(k_{x}^{2}-k_{y}^{2}\right)$ and $\left\langle|\eta(\hat{\mathbf{k}})|^{2}\right\rangle=$ 1 , the quadratic part of the MGL functional is

$$
\begin{aligned}
\mathcal{F}= & \Psi^{*}\left\{\alpha_{d}+\pi N_{0}\left[\frac{K_{03}}{4}\left\langle|\eta(\hat{\mathbf{k}})|^{2}(\mathbf{v} \cdot \boldsymbol{\Pi})^{2}\right\rangle\right.\right. \\
& -\frac{K_{05}}{16}\left\langle|\eta(\hat{\mathbf{k}})|^{2}(\mathbf{v} \cdot \boldsymbol{\Pi})^{4}\right\rangle \\
& \left.\left.-\frac{K_{15}}{32 \tau}\left\langle\eta(\hat{\mathbf{k}})^{*}(\mathbf{v} \cdot \boldsymbol{\Pi})^{2}\right\rangle\left\langle\eta(\hat{\mathbf{k}})(\mathbf{v} \cdot \boldsymbol{\Pi})^{2}\right\rangle\right]\right\} \Psi .
\end{aligned}
$$

*Also at Institut Universitaire de France, Paris, France.

${ }^{1}$ Y. Matsuda and H. Shimahara, J. Phys. Soc. Jpn. 76, 051005 (2007).

${ }^{2}$ P. Fulde and R. A. Ferrell, Phys. Rev. 135, 1550 (1964).

${ }^{3}$ A. I. Larkin and Yu. N. Ovchinnikov, Zh. Eksp. Teor. Fiz. 47, 1136 (1964) [Sov. Phys. JETP 20, 762 (1965)].

${ }^{4}$ L. G. Aslamazov, Zh. Eksp. Teor. Fiz. 55, 1477 (1968) [Sov. Phys. JETP 28, 773 (1969)].

${ }^{5}$ A. B. Vorontsov, I. Vekhter, and M. J. Graf, Phys. Rev. B 78, 180505 (2008).

${ }^{6}$ J. P. Brison, N. Keller, P. Lejoy, A. Huxley, L. Schmidt, A. Buzdin, N. R. Bernhoeft, I. Mineev, A. N. Stepanov, J. Flouquet, D. Jaccard, S. R. Julian, and G. G. Lonzarich, Physica B 199, 70 (1994).

${ }^{7}$ A. Buzdin, Y. Matsuda, and T. Shibauchi, Europhys. Lett. 80, 67004 (2007).

${ }^{8}$ A. I. Buzdin and L. N. Bulaevskii, Sov. Phys. Usp. 27, 830 (1984) [Usp. Fiz. Nauk 144, 415 (1984)].

${ }^{9}$ The Physics of Organic Superconductors and Conductors, edited by A. G. Lebed (Springer, Berlin, 2008).

${ }^{10}$ S. Uji, H. Shinagawa, T. Terashima, T. Yakabe, Y. Terai, M. Tokumoto, A. Kobayashi, H. Tanaka, and H. Kobayashi, Nature (London) 410, 908 (2001).

${ }^{11}$ K. Izawa, H. Yamaguchi, T. Sasaki, and Y. Matsuda, Phys. Rev. Lett. 88, 027002 (2001).

${ }^{12}$ J. Singleton, J. A. Symington, M.-S. Nam, A. Ardavan, M. Kurmoo, and P. Day, J. Phys.: Condens. Matter 12, L641 (2000).

${ }^{13}$ L. W. Gruenberg and L. Gunther, Phys. Rev. Lett. 16, 996 (1966).

${ }^{14}$ D. Saint-James, G. Sarma, and E. J. Thomas, Type II Superconductivity (Pergamon, New York, 1969).

${ }^{15}$ A. I. Buzdin and J. P. Brison, Phys. Lett. A 218, 359 (1996).

${ }^{16}$ J. P. Brison, N. Keller, A. Vernière, P. Lejay, L. Schmidt, A. Buzdin, J. Flouquet, S. R. Julian, and G. G. Lonzarich, Physica C 250, 128 (1995).
${ }^{17}$ D. Denisov, A. Buzdin, and H. Shimahara, Phys. Rev. B 79, 064506 (2009).

${ }^{18}$ A. I. Buzdin and H. Kachkachi, Phys. Lett. A 225, 341 (1997).

${ }^{19}$ H. Shimahara, Phys. Rev. B 80, 214512 (2009).

${ }^{20}$ A. I. Buzdin and J. P. Brison, Europhys. Lett. 35, 707 (1996).

${ }^{21}$ H. Shimahara and D. Rainer, J. Phys. Soc. Jpn. 66, 3591 (1997).

${ }^{22}$ M. Houzet and A. Buzdin, Europhys. Lett. 50, 375 (2000).

${ }^{23}$ U. Klein, D. Rainer, and H. Shimahara, J. Low Temp. Phys. 118, 91 (2000).

${ }^{24}$ U. Klein, Phys. Rev. B 69, 134518 (2004).

${ }^{25}$ S. Manalo and U. Klein, Phys. Rev. B 65, 144510 (2002).

${ }^{26}$ H. Adachi and R. Ikeda, Phys. Rev. B 68, 184510 (2003).

${ }^{27}$ F. Konschelle, J. Cayssol, and A. Buzdin, Europhys. Lett. 79, 67001 (2007).

${ }^{28}$ F. Konschelle, J. Cayssol, and A. I. Buzdin, Phys. Rev. B 79, 224526 (2009).

${ }^{29}$ A. A. Abrikosov, Fundamentals of the Theory of Metals (Elsevier, Netherlands, 1988).

${ }^{30}$ M. Houzet and V. P. Mineev, Phys. Rev. B 74, 144522 (2006).

${ }^{31}$ T. Maehira, T. Hotta, K. Ueda, and A. Hasegawa, J. Phys. Soc. Jpn. 72, 854 (2003)

${ }^{32}$ M. Houzet, Y. Meurdesoif, O. Coste, and A. Buzdin, Physica C 316, 89 (1999).

${ }^{33}$ S. K. Goh, Y. Mizukami, H. Shishido, D. Watanabe, S. Yasumoto, M. Shimozawa, M. Yamashita, T. Terashima, Y. Yanase, T. Shibauchi, A. I. Buzdin, and Y. Matsuda, Phys. Rev. Lett. 109, 157006 (2012)

${ }^{34}$ L. N. Bulaevskii, Zh. Eksp. Teor. Fiz. 65, 1278 (1973) [Sov. Phys. JETP 38, 634 (1974)].

${ }^{35}$ S. Uji, T. Terashima, M. Nishimura, Y. Takahide, T. Konoike, K. Enomoto, H. Cui, H. Kobayashi, A. Kobayashi, H. Tanaka, M. Tokumoto, E. S. Choi, T. Tokumoto, D. Graf, and J. S. Brooks, Phys. Rev. Lett. 97, 157001 (2006). 